Check for updates

Cite this: RSC Adv., 2021, 11, 33835

Received 13th June 2021

Accepted 24th September 2021

DOI: $10.1039 / d 1 r a 04573 c$

rsc.li/rsc-advances

\title{
Towards conductive hydrogels in e-skins: a review on rational design and recent developments
}

\begin{abstract}
Chujia Li iD *
Over the past decades, electronic skins (e-skins) have attracted significant attention owing to their feasibility of applications in health monitoring, motion detection, and entertainment. As a class of soft materials, conductive hydrogels feature biocompatibility, stretchability, adhesiveness, and self-healing properties, making them one of the most important candidates for high-performance e-skins. However, profound challenges remain for achieving the combination of superior mechanical strength and conductivity of conductive hydrogels simultaneously without sacrificing their multifunctionalities. Herein, a framework for rational designs to fabricate conductive hydrogels are proposed, including the fundamental strategies of copolymerization, doping, and self-assembly. In addition, we provide a comprehensive analysis of their merits and demerits when the conductive hydrogels are fabricated in different ways. Furthermore, the recent progress and future perspective for conductive hydrogels in terms of electronic skins are highlighted.
\end{abstract}

\section{Introduction}

Electronic skins (e-skins) have become an attractive topic in modern society since they brought utmost convenience to monitor the pulse, heartbeats, motion, and other activities of human beings. ${ }^{1-4}$ Owing to the responsive properties, the circuit systems of e-skins can transform the environmental stimuli (e.g., temperature, pressure, strain, and light) into electrical signals and give appropriate responses, shining a light on the usage in biomedical and energy storage industries. ${ }^{3,5-10}$ So far, many works on fabricating e-skins have focused on inorganic materials, including graphene, ${ }^{11-13}$ semiconductors, ${ }^{14-18}$ and alginate-based materials. ${ }^{19}$ Although they exhibit outstanding conductivity and mechanical properties, it remains a challenge to achieve softness, self-healing properties, and biocompatibility, which are critical factors for a high-performance eskin. ${ }^{20,21}$ Conductive hydrogel is one of the most popular alternative candidates to tackle these issues by virtue of the excellent electrical conductivity and flexibility, ${ }^{22-25}$ which highly matches the demands for fabricating flexible electronics, especially the critical conditions of e-skins.

Recent advances of conductive hydrogels have attracted a lot of attention on e-skins owing to their stretchability, ${ }^{16-18}$ sensitivity, ${ }^{28,29}$ self-repairing capacity, ${ }^{30-32}$ flexibility,,$^{26,27,33}$ and excellent compatibility. ${ }^{34-36}$ However, in practical applications of e-skins, traditional hydrogels are not robust enough to sustain cyclic loading and impact strength. ${ }^{6}$ There is an urgent need to improve the

Queen Mary University of London Engineering School, Northwestern Polytechnical University, Xi'an, Shaanxi Province 710072, China. E-mail: chujiali@mail.nwpu. edu.cn mechanical strength and conductivity of hydrogels without impairing their own features, such as stimulus-responsive compatibility, adhesiveness, and biocompatibility. Various synthesis methodologies have been proposed to develop advanced conductive hydrogels to address such challenges, including copolymerization, doping, and self-assembly (Fig. 1). ${ }^{23,37,38}$

Among such important techniques, copolymerization is an effective and straightforward strategy to modulate the

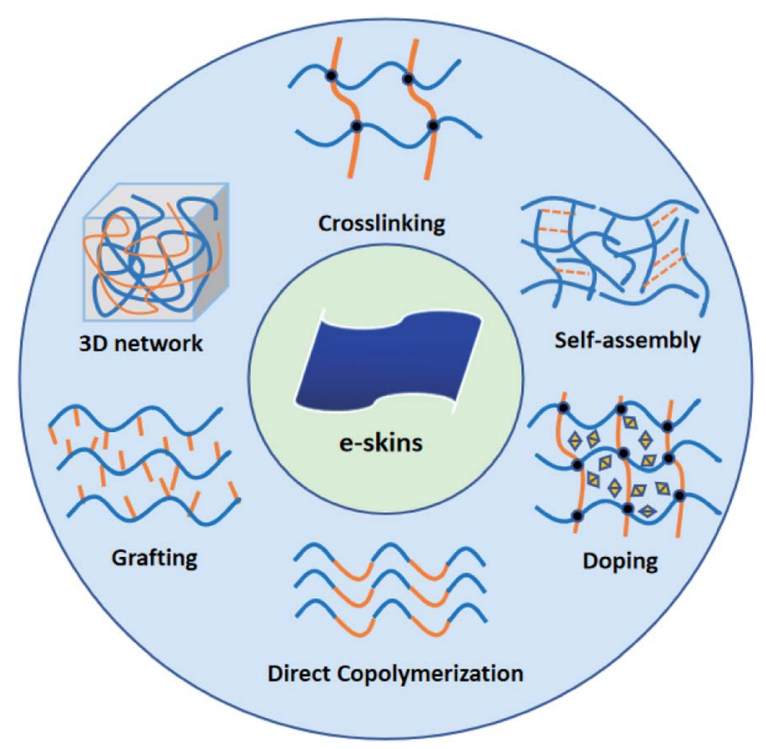

Fig. 1 Illustration of structural designs and methodologies to fabricate conductive hydrogels in the application of e-skins. 
proportions of different monomers via direct copolymerization reaction, ${ }^{39}$ or modify the functional groups to realize specific properties and introduce crosslink networks by grafting. ${ }^{\mathbf{4 0 - 4 2}}$ Representative systems for copolymerization are conductive polymers include polyaniline (PANI) and polypyrrole (PPy), which are attributed to their excellent conductivity and biocompatibility, or hydrophilic chains including poly( $N$-vinylpyrrolidone), poly(ethylene glycol), and polyacrylic acid, to endow the hydrogels with self-healing and adhesiveness. ${ }^{23,41-43}$ Such strategy is easy to be processed, but a serious concern is that the conductive stability and mechanical strength (i.e., in the range of tissues and organs) of hydrogels are difficult to be accurately controlled since these properties are tightly dependent on the amounts and features of each monomer. ${ }^{23}$

Consequently, it is arduous to achieve accurate control during the polymerization process. To tackle this issue, doping is selected as a facile method to adjust the conductivity and mechanical strength of hydrogels directly by simply incorporating conductive charges, acids, or polymer chains. ${ }^{26,33,37,44-48}$ Also, the stability of the system can be enhanced by forming gelated nanostructures in hydrogels. ${ }^{37,49}$ It is generally accepted that the conductive PPy, PANI, and phytic acid (PA) can be doped into the hydrogel structure to enhance the conductivity, and the porous microstructure or crosslinking network can be generated to endow the hydrogels with high stretchability. ${ }^{\mathbf{5 0 , 5 1}}$

Furthermore, self-assembly is regarded as another novel and advanced strategy to fabricate conductive hydrogels with selfhealing properties due to the existence of weaker interactions among polymer chains, including $\mathrm{p}-\mathrm{p}$ stacking, ${ }^{5-55}$ van der Waals forces, ${ }^{56}$ hydrophobic interactions, ${ }^{57,58}$ hydrogen bonding, ${ }^{59}$ and electrostatic interactions, ${ }^{60,61}$ which all effectively drive the formation of hydrogels. However, it should be noted that the stability of self-assembled hydrogels is still limited because they may largely be influenced by the external environment. ${ }^{62,63}$ Thus, further effort is required to develop a hydrogel system that is sufficiently stable and efficient for practical use in e-skins.

Herein, we propose a theoretical framework of methodologies to summarize the recent progress in high-performance conductive hydrogels for flexible electronics and further provide novel insights about the rational design of advanced hydrogels based on their structural designs and the chemical interactions in the polymer networks. We review the feasible methodologies of synthesizing conductive hydrogels, present the major advantages for each approach, and highlight their bright perspectives in the applications of flexible electronic devices, especially for their utilization and great advantages for e-skins. This review aims to construct a clear theoretical framework to illustrate the main fabrication approaches for conductive hydrogels and provide novel insights to develop the advanced strategies of hydrogel fabrication, while their promising future on the potential usage of e-skins is further clarified and discussed.

\section{Copolymerization}

Copolymerization reaction is a quintessential methodology to generate conductive hydrogels by combining the unique properties in different types of monomers, polymer chains, or components. ${ }^{64,65}$ Among all the copolymerization strategies, direct copolymerization and grafting are two widely utilized approaches to fabricate conductive hydrogels through copolymerization, realizing multifunctional features of hydrogels, such as conductivity, stretchability, stability, self-healing property, and biocompatibility. ${ }^{34,35,66-81}$ However, the majority of hydrogels are restricted by the poor mechanical strength, ${ }^{\mathbf{2}}$ leading to insufficient stability and efficiency for practical utilization. To tackle the limitations on structural robustness, incorporation of high-strength fillers and constructing crosslinked structures are designed during fabrication. ${ }^{34,70,71,78,80}$ By optimizing conductive hydrogels featuring superior strength and conductivity, they are of paramount importance in the applications of e-skins or wearable electronics.

\subsection{Direct copolymerization}

Direct copolymerization is of great significance to fabricate conductive hydrogels by simply mixing monomers with different ratios or adding them into hydrogel precursors, followed by feeding a gelator or changing the external environments to activate the reaction. ${ }^{83}$ These conductive hydrogels can be applied to generate multifunctional e-skins or flexible electronic devices. ${ }^{66-73} \mathrm{~A}$ facile methodology of direct copolymerization for conductive hydrogels is to control the content of monomers in the copolymers in order to realize the specific properties of hydrogels. For example, Jiang et al. established a mechanical-tunable conductive hydrogel by copolymerizing hydrophobic HEMA (hydroxyethyl methacrylate) and AA (acrylic acid), catering to the different requirements of mechanical properties of hydrogels. ${ }^{66}$ Owing to the adjustable mechanical and conductive capacity, ${ }^{66}$ such a hydrogel has great potential to be applied in flexible electronic wearable devices and sensor usages.

To enhance the mechanical strength and conductivity, copolymer hydrogel is interpenetrated with CNT. In the past decade, several works have utilized this strategy to further optimize the performance of hydrogels. ${ }^{\mathbf{8 4}, \mathbf{8 5}}$ However, during the chemical treatment of CNT, its p-conjugation can be disrupted, affecting the performance of CNT on the electrical conductivity. ${ }^{86}$ A study reported by Li et al. provided a facile approach to tackle this challenge by interpenetrating single-walled carbon nanotubes (SWCNTs) into a pyrene-and-borate-modified $\mathrm{P}$ (DMA-co-APB-co-ABA) hydrogel composite, and such selfhealable, electrical conductive hydrogels suggest a desirable future in e-skins. ${ }^{72}$ Nanosheets or nanocomposites are possible fillers for fabricating conductive hydrogels. Wang and coauthors intersected XLG nanosheets into SBMA-HEMA copolymers to fabricate a conductive nanocomposite hydrogel with self-healing ability, which can be applied to synthesize skin strain sensors. ${ }^{68}$ Notably, the XLT can adsorb zwitterions, generating reversible physical interaction among chains and improving mechanical properties. ${ }^{68}$

Besides adding CNT or nanosheet fillers, introducing conductive metal ions is also an excellent strategy to endow the system with high conductivity. ${ }^{65,69,71}$ Tan et al. reported 
a thermo-sensitive hydrogel by constructing the chemical and physical interaction between $\mathrm{Al}^{3+}$ and P(DMAPS-co-AA) copolymer. ${ }^{71}$ It reveals a promising application in electrocardiogram monitoring due to its enhanced conductivity and mechanical strength. ${ }^{71}$ Moreover, the metal ions can also form strong coordinate bonds with other polymer chains, improving the self-healing and mechanical properties of hydrogels. ${ }^{65,87} \mathrm{~A}$ typical example is the study reported by Das and co-authors, who proposed a poly(4-styrene sulfonate- $c o$-methyl-uracilimidazolium) chloride (PSS-MUI), followed by adding ferric ions as the second crosslinker into the gelatin system (Fig. 2a1 and a2). ${ }^{69}$ The as-prepared double-network hydrogel can be applied in wearable electronic devices (Fig. 2a3). ${ }^{69}$

Another method to fabricate a conductive hydrogel with high mechanical properties via direct copolymerization is to build physical and chemical crosslinks between different polymer chains, combining the advantages of each polymer. Wang et al. developed a tough, conductive hydrogel by adding polyaniline (PANI) chains into the biocompatible P(AAm-co-HEMA) copolymer crosslink network, which is promising in flexible electronic devices by virtue of its high sensitivity and linearity in the presence of large strains (Fig. $2 \mathrm{~b} 1$ and b2). ${ }^{67}$ PAM is also

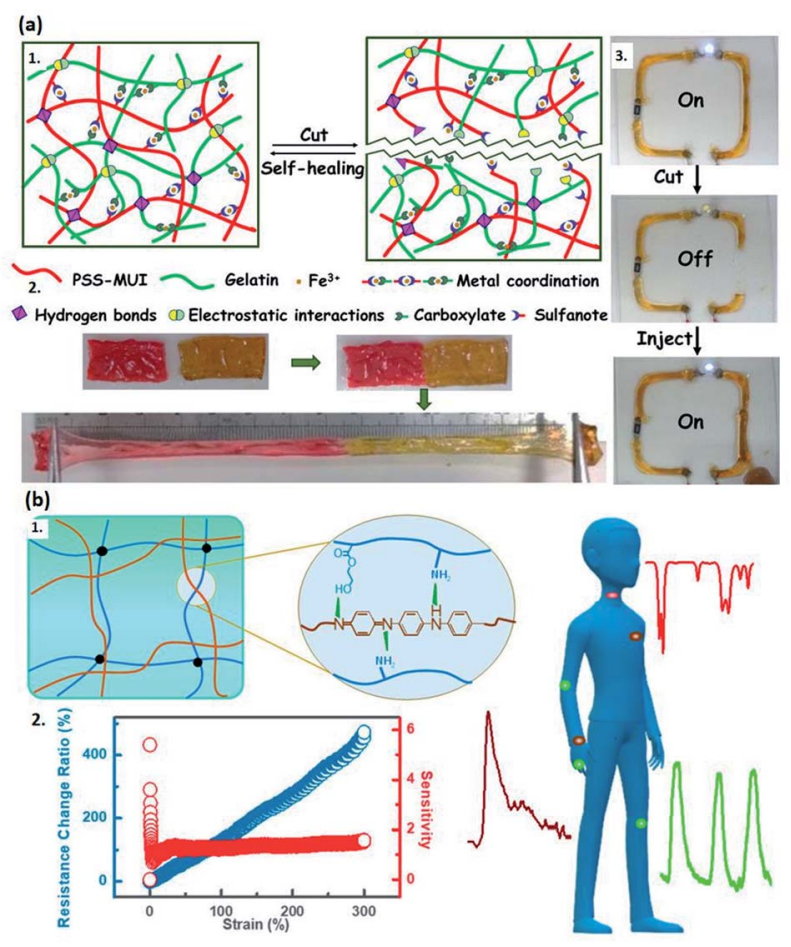

Fig. 2 Conductive hydrogels fabricated via copolymerization approach. (a) Schematic representation of $\mathrm{Fe}^{3+}$-based conductive hydrogel. (a1) The mechanism of self-healing property. (a2) The stretchability of PSUGF-2 hydrogel strips after healing at room temperature for $120 \mathrm{~min}$. (a3) The conductivity test for a flexible electric circuit assembled by PSUGF-2 hydrogel. (Adapted with permission from ref. ${ }^{69}$. Copyright 2020, American Chemical Society). (b) Schematic illustration of (b1) the generated PANI/P(AAm-CO-HEMA) hydrogels, (b2) the relationship between resistance change ratio and tensile strain, and (b3) the expected application in e-skins. (Adapted with permission from ref. ${ }^{67}$. Copyright 2018, American Chemical Society). frequently introduced into the hydrogel systems to form crosslinks due to its superior flexibility and stretchability. ${ }^{88,89}$ Long et al. reported a strategy to fabricate a PDA-PAM hydrogel crosslinking network composed of self-polymerized PDA, AMBIS copolymers, and tetramethylethylenediamine (TMEDA). ${ }^{73}$ The as-prepared hydrogels are further synthesized as an electrode in a sandwich configuration (PP-TENG). ${ }^{73}$ Similar methodology is also expected to be utilized to develop e-skins due to the excellent stretchability, self-repairing ability, and conductivity. To achieve more accurate control of the crosslinking network growth, Liu and co-authors utilized PEG-Gly as a template, where AM and AA monomers can be copolymerized, generating a PEG/PAMAA conductive hydrogel. ${ }^{70}$ Owing to the self-healing property and high sensitivity, it would have more hopeful prospects in flexible devices. ${ }^{70}$

Various works demonstrate the applications of conductive hydrogels prepared by direct copolymerization. ${ }^{90-92}$ Due to the superior stretchability of conductive hydrogels, they are typically chosen to assemble flexible strain sensors. ${ }^{93}$ For example, Liu et al. fabricated a wearable sweaty hydrogel strain sensor based on DNA-inspired block polymers, which exhibits desirable sensitivity to human motion, including head nodding, speaking, and joint bending. ${ }^{92}$ Notably, it is highly adhesive to sweat and water at different temperatures, indicating suitability to be applied in extreme environments. ${ }^{92}$ Solid-state supercapacitor is another example for e-skins, where hydrogels play a significant role in the conductive electrode owing to the high capacitive energy density and scalable processibility. ${ }^{94} \mathrm{Li}$ et al. developed a flexible solid-state supercapacitor, which comprises polyaniline-polyvinyl alcohol hydrogel (PPH) as an electrode. ${ }^{95}$ It has superior specific capacitance, mechanical properties, and cyclic stability compared to other supercapacitors, ${ }^{95}$ leading it to be a promising candidate to integrate with wearable textiles and form flexible sensors for health monitoring. ${ }^{96}$ Apart from smart wearable sensors for human monitoring or motion detection, conductive hydrogels also show great potential in the robotic field to detect real-time actuations of robots. ${ }^{97}$ Yang and coauthors reported a high-performance rGO/poly(AMPS-co-AAm) nanocomposite hydrogel, which shows significant reversible and repeatable electro-response under cyclic applications of the electric field. ${ }^{90}$ Such hydrogels can be regarded as an actuator for cantilevers and grippers on soft robots. ${ }^{90}$

\subsection{Grafting modification}

Grafting is another critical strategy to synthesize conductive hydrogels on account of the porous structure in the hydrogel network, enabling the hydrogels to become more flexible and softer. Compared with traditional copolymerization approaches, grafting can further improve the crosslinking density and secondary interactions between polymer chains. ${ }^{74}$ For instance, Chen et al. fabricated a cellulose-based conductive hydrogel grafted with acrylonitrile and acrylamide copolymers, which can create secondary interactions between copolymer chains, improving the ultra-stretchability, toughness, and antifreezing properties of the hydrogel. ${ }^{74}$ Such conductive hydrogels can be used to produce flexible strain sensors. ${ }^{74}$ 
Grafting conductive oligomers, polymer chains, and nanocomposites is the common strategy to enhance the mechanical strength and conductivity of hydrogels..$^{34,76,77,81}$ It is well established that aniline tetramer (AT) and PPy are desirable grafting agents owing to their high conductivity and biocompatibility. ${ }^{98,99}$ Zhao et al. developed a conductive injectable hydrogel by a thermalgelling approach. ${ }^{76}$ Specifically, AT is grafted on poly(caprolactone)-poly(ethyleneglycol)-poly(caprolactone) copolymer and form amphiphilic AT-PCEC hydrogel with excellent biocompatibility, ${ }^{76}$ which is expected to be an excellent candidate for eskins. Dong and co-authors also utilized AT to prepare a biodegradable injectable hydrogel, which consists of chitosan-graft-AT and PEG-DA copolymers and performs excellent self-repairing ability and antibacterial activity. This hydrogel is not only an ideal material to fabricate vehicles as cell delivery carriers, ${ }^{77}$ but also presents a wide range of possibilities for further development of e-skins due to its biocompatibility and conductivity. ${ }^{77}$ To further enhance the biocompatibility and self-healing ability, Wang et al. designed a gelatin-based injectable hydrogel by grafting conductive PPy on the MA-grafted gelatin hydrogel network and incorporated ferric ions into the system to enhance the conductivity. ${ }^{\mathbf{8 1}}$ The hydrogel exhibits self-healing properties, ${ }^{81}$ making it a suitable material for e-skins. Besides grafting conductive polymer chains, Mandal and co-authors creatively utilized poly(acryloyl hydrazide)silver nanocomposite reinforcements with different sizes, followed by grafting it into PAMAS hydrogel matrix. The constructed ionic linkages play a significant role in improving the mechanical strength of the hydrogel (Fig. 3a1). ${ }^{34}$ The as-prepared hydrogel shows high conductivity, toughness, and stretchability (Fig. 3a2), which can be applied in flexible device applications such as ECG monitoring (Fig. 3a3), ${ }^{34}$ raising the prospects in electronic skins.

During the grafting process, rGO or GO is typically incorporated in the hydrogel system to reinforce the grafted structure. ${ }^{35,75,78,79}$ Dai and co-authors filled GO into the acrylic acid-acrylamide (AA$\mathrm{AM}$ ) grafted cellulose hydrogel, which is highly responsive to $\mathrm{pH}$, salt, and organic solvent, making it a good candidate in the drug delivery system. ${ }^{75}$ Their work might offer a new avenue for flexible electronic devices. Similarly, Liang et al. developed a P(QCSG- $g$ GM) copolymer, followed by adding GO fillers. ${ }^{79}$ The synthesized injectable hydrogel is anti-bacterial, conductive, and photothermal responsive. $^{79}$ As a filler, GO endows the hydrogels with high conductivity and photothermal property, ${ }^{79}$ and it could be a desirable material for flexible electronic devices. On the other hand, rGO is also widely used to enhance the mechanical properties of hydrogels, and it exhibits better gel properties than GO. ${ }^{80}$ Liang et al. reported an HA-DA/rGO hydrogel by grafting hyaluronic acid onto dopamine, followed by incorporating rGO in the $\mathrm{H}_{2} \mathrm{O}_{2} / \mathrm{HPR}$ system. $^{35}$ The self-healing, adhesive hydrogel has excellent injectability, satisfying the requirements in wound dressing, ${ }^{35}$ realizing a bright future in e-skins and wearable electronic devices. Likewise, Jafarigol et al. developed a carboxymethyl cellulose-based conductive hydrogel, which is grafted by AM-AC copolymer, and its swelling behavior is further investigated by adding rGO with different ratios (Fig. 3b). ${ }^{78}$ Such a hydrogel exhibits heat-resistant, electro-conductivity, and excellent mechanical properties, opening the door to new possibilities in flexible electronics and soft energy storage systems. ${ }^{78}$ (a)

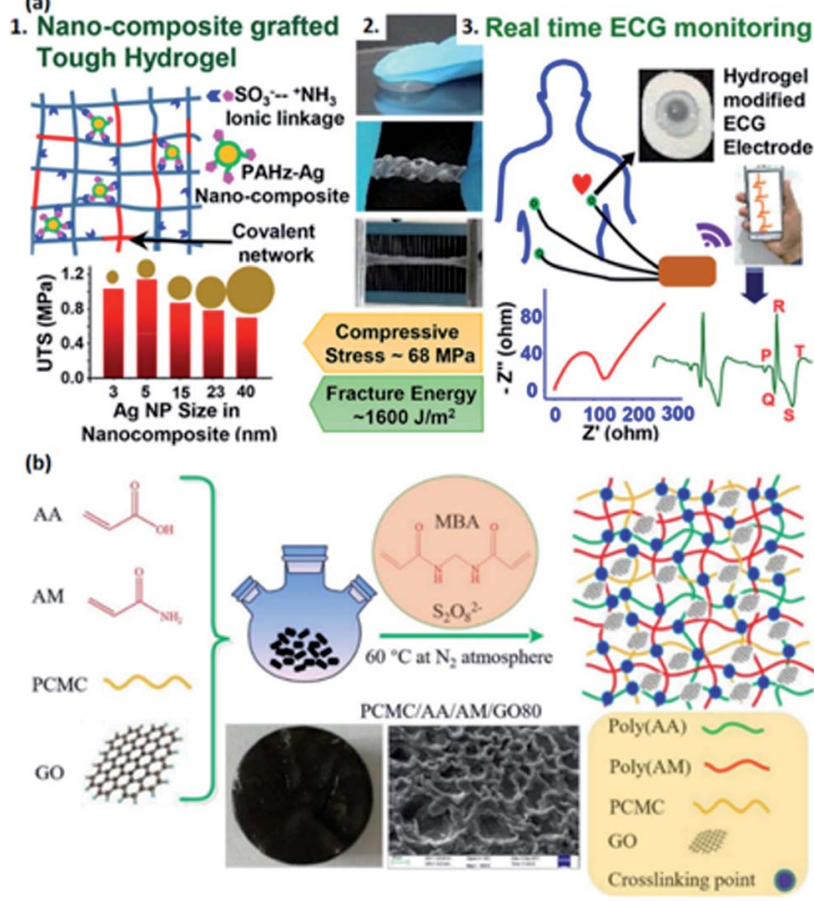

Fig. 3 The representation of conductive hydrogels fabricated by grafting. (a) Nanocomposite-grafted PAMAS conductive hydrogel with (a1) the covalent network and ionic linkage, and (a2) the stretchability and toughness have improved. (a3) Modification on the ECG electrode for monitoring. (Adapted with permission from ref. ${ }^{34}$. Copyright 2020, American Chemical Society). (b) Schematic representation for fabricating AA-AM-grafted cellulose hydrogel reinforced by the GO fillers. (Adapted with permission from ref. ${ }^{78}$. Copyright 2019, Elsevier).

Grafting modification is also a strategy that provides effective solutions to the applications of e-skins for conductive hydrogels. ${ }^{\mathbf{1 0 0 , 1 0 1}}$ Strain sensors are widely fabricated using conductive hydrogels to optimize the sensitivity and stretchability of conventional sensors. Chen and co-authors developed a conductive cellulose hydrogel grafted by acrylonitrile and acrylamide copolymers. The hydrogel was further demonstrated as a suitable candidate for wearable strain sensors by virtue of its superior sensitivity and stability, which is desirable for human healthcare detections or motion monitoring. ${ }^{\mathbf{1 0 0}}$ On the other hand, the improvement in crosslinking density by grafting modification enables the hydrogels to be more flexible without sacrificing their stability and mechanical strength ${ }^{74}$ indicating a future perspective in the electrode, especially for supercapacitors or batteries. For instance, Huang et al. reported a PAM-grafted VSNPs conductive hydrogel film, which was utilized as an electrolyte in a supercapacitor, exhibiting great sensitivity and compressibility. ${ }^{74}$ Since few supercapacitors with super-compressive properties have been investigated, ${ }^{74}$ this research paves a new road on compressive energy devices and robotic systems.

\section{Doping}

As one of the most important strategies to modify conductive hydrogels on account of the three-dimensional tunable network, doping has captured a huge amount of interest 
already. ${ }^{37}$ The dopants play a significant role in enhancing the conductivity, mechanical strength, and stability of conductive hydrogels, ${ }^{27,31,33,44-48,102-110}$ and polymer dopants have gained more popularity than other dopants such as acids, additives, small molecules, or metal salts. The introduction of dopants to conductive hydrogels makes them easier to control for physical and chemical properties as well as morphology compared to the traditional conductive hydrogels. ${ }^{37}$ Hence, the doped conductive hydrogels are great candidates to synthesize e-skins.

\subsection{Polymer dopants}

Polymer dopant is of great importance in modifying the desirable properties (conductivity, flexibility, biocompatibility, toughness, etc.) of hydrogels during the fabrication process. Representative polymer dopants such as PPy, PANI, and poly(3,4-ethylenedioxythiophene):polystyrenesulfonate

(PEDOT:PSS) for conductive hydrogel synthesis are widely used because they overcome the poor conductivity and mechanical properties of traditional hydrogels and endows special features with promising utilization in energy storage systems, biomaterials, and sensors. ${ }^{31,47,48,107-110}$

PPy, as a traditional dopant, can enhance the electrical conductivity and compatibility of hydrogels, ${ }^{107}$ expanding the possibilities of hydrogels for the development of e-skins or soft sensor applications. $\mathrm{Bu}$ et al. reported sodium alginate and carboxymethyl chitosan-based conductive hydrogel using PPy as a dopant, which exhibits biocompatibility and may achieve controllable conductivity and mechanical properties by adjusting the content of dopants. ${ }^{\mathbf{1 1 0}}$ Therefore, this hydrogel is an ideal material for fabricating flexible electronics. Recently, Zhao et al. synthesized a tough and stretchable hydrogel based on chitosan network, PAA, and $\mathrm{Fe}^{3+}$, followed by doping PPy particles into the pre-hydrogel network, and thus the conductivity of the hydrogel can be enhanced (Fig. 4a1). ${ }^{107}$ The asprepared hydrogel has excellent self-healing properties, which can be embedded into soft electronics or sensors (Fig. 4a2). ${ }^{107}$ By contrast, PPy can also be doped by other polymers to endow the hydrogel with other suitable properties. Han and co-authors developed a conductive hydrogel by incorporating dopant polydopamine into PPy fibers. ${ }^{47}$ The hydrophilicity of the polymer network enables it to further interweave with polyacrylamide to form PDA-PPy-PAM hydrogel. ${ }^{47}$ Notably, the hydrogel has a light-transmitting property, having wide applications such as transparent flexible electronics, biosignal detection, etc. ${ }^{47}$

Doping a polymer blend is also a strategy to synthesize conductive hydrogels such as PEDOT:PSS. ${ }^{109}$ As an example, Wu and co-authors fabricated a poly( $\mathrm{N}$-acryloyl glycinamide- $\mathrm{Co}-2$ acrylamide-2-methylpropanesulfonic) conductive hydrogel, followed by doping with poly(3,4-ethylenedioxythiophene)-poly (styrenesulfonate) (PEDOT/PSS), featuring relatively high conductivity. ${ }^{109}$ The as-prepared hydrogel has excellent selfrepairability, thermal processability, and stretchability, enabling the utilization in sensors and biomaterials. ${ }^{109}$ Taking advantage of the water-dispersive property of PEDOT:PSS as a dopant, Spencer and co-authors also developed a conductive hydrogel based on gelatin methacryloyl (GelMA) doped by

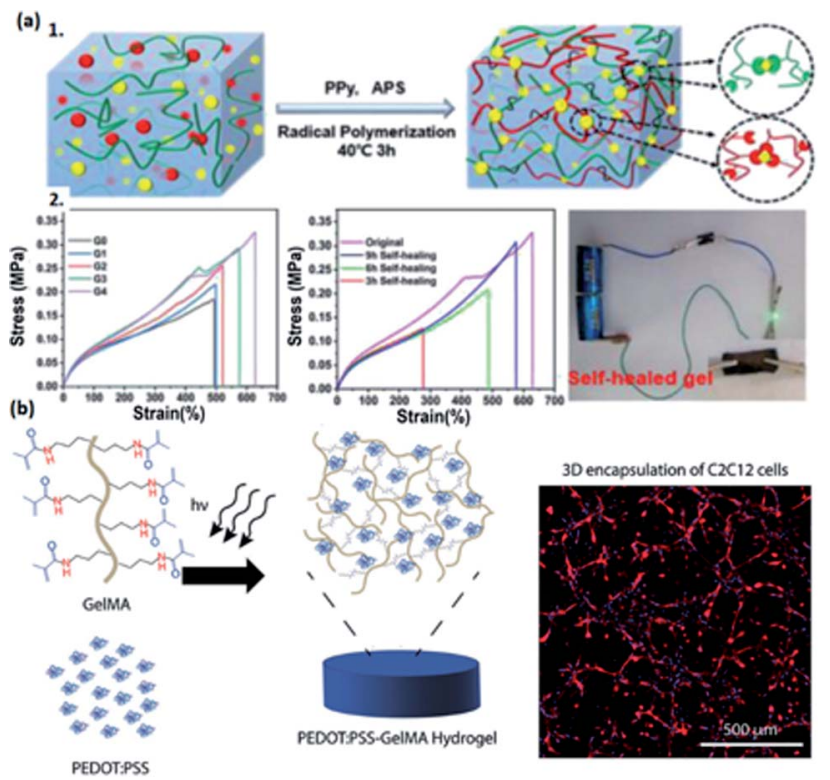

Fig. 4 The illustration of representative conductive hydrogels with the modification of different types of polymer dopants. (a1) The mechanism of fabricating CS/PAA/PPy/Fe(III) hydrogels. (a2) Influence of selfhealing time and Fe(III) on the mechanical properties of hydrogels and the conductive behavior of self-healed hydrogels. (Adapted with permission from ref. ${ }^{107}$. Copyright 2021, American Chemical Society). (b) Fabrication of PEDOT:PSS-GelMA hydrogel and actin/DAPI image from $\mathrm{C} 2 \mathrm{C} 12$ cells encapsulated in 0.1\% PEDOT:PSS over 5 days of culture (scale bars $=500 \mu \mathrm{m}$ ). (Adapted with permission from ref. ${ }^{48}$. Copyright 2018, American Chemical Society).

PEDOT:PSS, exhibiting electrical-tunability, photo-sensitive capacity, and such hydrogels can be applied in biomaterial manufacturing, ${ }^{48}$ having a promising future in e-skins and flexible electronic devices (Fig. 4b).

The studies about polymer-doped conductive hydrogels reveal the possible applications of e-skins, including flexible strain sensors, bioelectronics, and electrodes. ${ }^{111,112}$ One interesting example is the hydrogel-based sensors and bioelectrodes developed by Han et al., which are fabricated by PDA-doped PPyPAM conductive hydrogels. ${ }^{111}$ They can detect body motion without extra aids and show superior tissue adhesiveness. ${ }^{111}$ Besides, the as-prepared hydrogels have UV shielding ability, ${ }^{\mathbf{1 1 1}}$ indicating a bright future in smart textiles. Similarly, Chen and co-authors fabricated a PANI/PSS hydrogel network and successfully assembled it into a strain sensor for accurate human movement detection, such as vocal vibration, wrist pulse, finger bending, etc. ${ }^{\mathbf{1 1 2}}$ Such hydrogel-based strain sensors can have diverse utilizations in either healthcare or daily monitoring.

PANI is another important dopant to improve the conductivity of hydrogels with a simple fabrication approach. ${ }^{31}$ Doping PANI into different gel systems can achieve widespread applications of hydrogels, depending on the features of the system being doped. Das and co-authors adopted a hybrid PANI-doped conductive hydrogel, which is crosslinked by folic acid and embedded with AgNPS nanoparticles to improve the conductivity and photo-responsive properties, realizing a promising 
application in the energy storage field (Fig. 5a). ${ }^{108}$ Likewise, Chakraborty et al. successfully doped polyaniline into $\mathrm{N}$-fluorenylmethoxycarbonyl diphenylalanine hydrogel, enhancing the conductivity and biocompatibility of the polymer, and the Fmoc-FF-PANI hydrogel has a promising future in sensing and biomedical fields. ${ }^{31}$ Such hydrogels could be used to synthesize flexible electronics. Moreover, PANI can also be combined with other strategies to prepare conductive hydrogels, such as doping by acid to enhance its conductivity, which will be discussed in the next section.

\subsection{Acid dopants}

Acid dopants are used to endow the hydrogels with high conductivity by tuning the concentrations of the dopant so that different concentrations of charge carriers can be achieved. ${ }^{\mathbf{1 0 3}}$ Consequently,

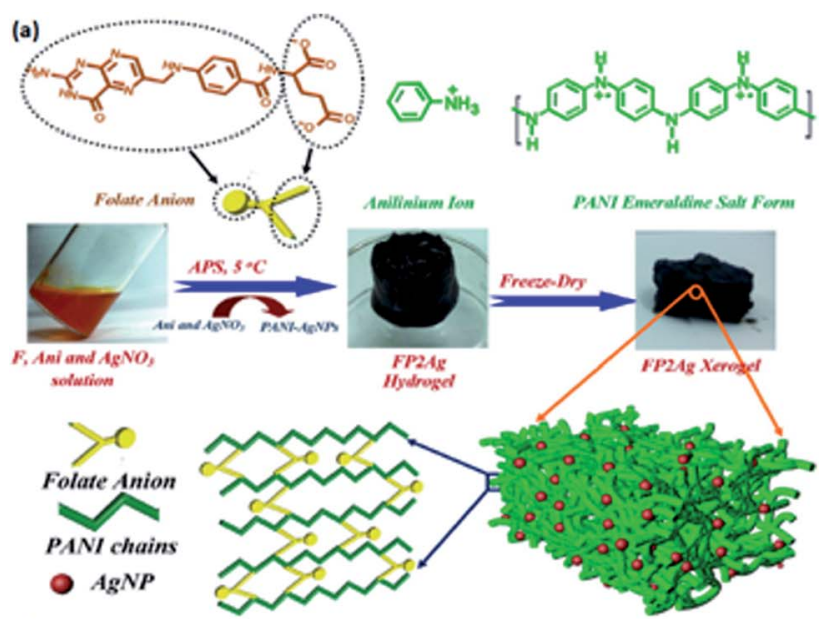

(b)

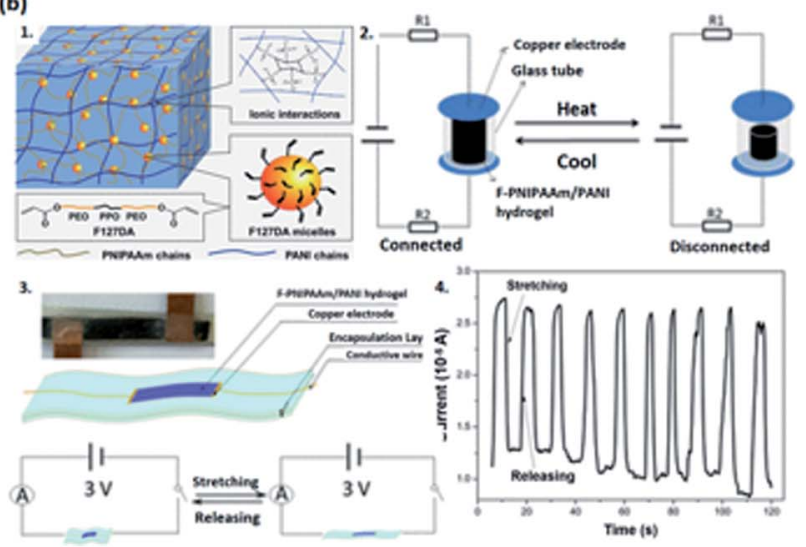

Fig. 5 PANI-based conductive hydrogels. (a) Illustration of PANIdoped AgNPS/Fe ${ }^{3+}$ hydrogels and the crosslinking network. (Adapted with permission from ref. ${ }^{108}$. Copyright 2018, American Chemical Society). (b) The representation of F-PNIPAAm/PANI hydrogels doped by PA. (b1) The network structure. (b2) The temperature alertor assembled by F-PNIPAAm/PANI hydrogels under cyclic heating and cooling. (b3) The configuration of a strain sensor based on-PNIPAAm/ PANI hydrogels when being stretched and released under $3 \mathrm{~V}$ (Adapted with permission from ref. ${ }^{33}$. Copyright 2018, American Chemical Society). (b4) The illustration of current response under $50 \%$ periodical tension strain of the strain sensor (Adapted with permission from ref. ${ }^{33}$. Copyright 2018, American Chemical Society). doping acid into the conductive polymer is a facile methodology to adopt an enhancement on the conductivity of hydrogels through protonation to achieve equilibrium interactions between polymer chains to endow good mechanical capability of hydrogels, ${ }^{37}$ and such acid-doped conductive hydrogels have widespread applications such as flexible sensors, energy storage devices, and electrocatalysts. . $3,44-46,102,103$

Phytic acid (PA) is selected to dope a conductive hydrogel (typically PANI or PPy network) to enhance its electrical conductivity, which has a bright future in e-skins and flexible electronics. ${ }^{3,44-46}$ Wang et al. adopted a hydrogel with high stretchability due to its dually synergetic network, followed by incorporating electro-conductive PANI doped with PA into thermal-sensitive poly( $N$-isopropylacrylamide) (PNIPAAm), forming a crosslinked network (Fig. 5b1). ${ }^{33}$ The as-prepared FPNIPAAm/PANI hydrogel has great potential in strain and temperature sensors (Fig. 5b2-b4). ${ }^{33}$ Similarly, Zhu and coauthors developed a hybrid conductive hydrogel by introducing PANI into polyion complex in the presence of PA as a dopant and crosslinker, which enhances the mechanical properties and conductivity of the hydrogel, and it is suitable for fabricating flexible electronics and strain sensors (Fig. 6a-c). ${ }^{44}$ Besides, PA can also be doped into PPy hydrogels; for example, $\mathrm{Hu}$ et al. doped phytic acid (PA) into conductive PPy, followed by coating with a carbon cloth to synthesize PA-PPy/CC hydrogel

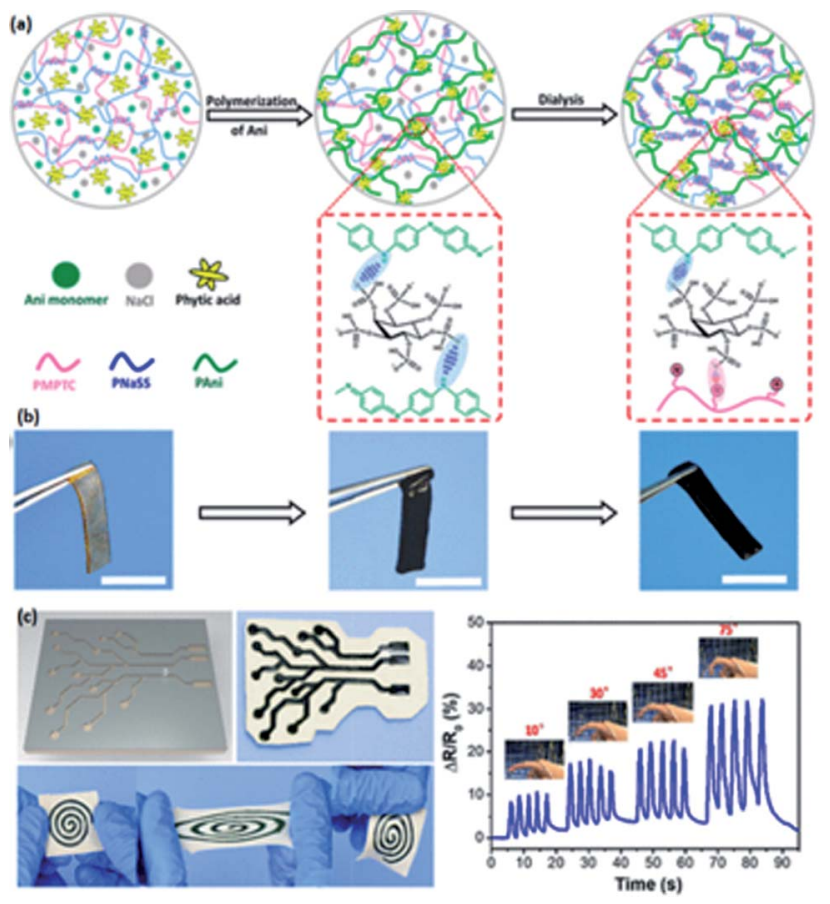

Fig. 6 Illustration of conductive hydrogels based on PANI. (a) Fabrication approach for PIC/PAni hybrid gels doped by PA. (b) The images for PIC/PAni hybrid gels under various mechanical states during the fabrication. (c) The recoverability of the generated PIC/PAni hydrogel, and the behavior of the PIC/PAni hydrogel sensor in response to different magnitudes of repetitive finger bending. (Adapted with permission from ref. ${ }^{44}$. Copyright 2018, American Chemical Society). 
with superhydrophilic properties. Such hydrogels can be used as electrocatalysts for oxygen evolution reactions (OER). ${ }^{45}$

Besides, other acid dopants are also useful in synthesizing conductive hydrogels, depending on the properties and features of each acid. Tannic acid is a suitable dopant for hydrogels in biomedical applications due to its high capability to improve both the mechanical strength and conductivity of hydrogels. ${ }^{113-116}$ For instance, Zhou and co-authors used tannic acid as a dopant and crosslinker to modify the PPy hydrogel backbone, which exhibits excellent conductivity. ${ }^{46}$ This biocompatible conductive polymer hydrogel can be applied in producing biomaterials. ${ }^{46}$ More recently, Zhou et al. synthesized a PAV/PANI hydrogel doped with TA, exhibiting suitable mechanical strength and conductivity by adjusting the concentrations of TA. ${ }^{102}$ Such hydrogels can be further applied to produce electrodes for pressure sensors owing to their excellent sensitivity and conductivity. ${ }^{102}$ On the other hand, since sulfuric acid contains high concentrations of protons, it could be used to control the ionic strength and water absorption ability of the hydrogel. ${ }^{103}$ Katzenberg et al. reported a facile method to synthesize polymer-electrolyte membranes, using conductive hydrogel as an electrolyte. ${ }^{103}$ Specifically, sulfuric acid is doped into a poly(acrylic acid)-poly(vinyl alcohol) network, increasing the conductivity of the system. ${ }^{103}$ Such homogenous, crosslinked structures are expected to be one of the most important alternative materials for e-skins.

Acid doping is a strategy that shows a powerful impact on the applications of electronic skins for conductive hydrogels. ${ }^{17-119}$ As for the Lithium Ion Battery (LIB) electrode, the conductive hydrogel framework is typically incorporated with various active materials and doped with acids to ensure the smooth transfer of electrons and ions. ${ }^{120,121}$ Shi and co-authors studied a 3D nanostructured PPy gel framework, which was employed as the binder and framework for the nanoparticle-based LIB electrode with superior capacity and conductivity. ${ }^{119}$ Furthermore, $\mathrm{Fe}_{3} \mathrm{O}_{4}$ nanoparticles were assembled into the gel framework to improve the electron and ion transport. ${ }^{119,121}$ Such an electrode is a promising candidate of anode material for the nextgeneration flexible LIBs. ${ }^{119}$ The second example is a piezoresistive sensor which was developed by Pan et al., which is composed of a PA-doped PPy hydrogel thin film with high elasticity and sensitivity, enabling the accurate detection of subtle variations of pressure, showing great potential in robotic and industrial detections. ${ }^{118}$ Besides, bioelectronics are widely developed using conductive hydrogels, where acid doping is typically used to enhance stability and conductivity. Zhang and co-authors reported an injectable, healable, and soft organic bioelectronics based on PEDOT:PSS conductive hydrogels, where 4-dodecylbenzenesulfonic acid (DBSA) as a dopant improved the ionic strength and protonation. ${ }^{117}$ Such bioelectronic can be potentially used as injectable conductors or further developed as organic electrochemical transistors (OECTs) for biomedical applications. ${ }^{117}$

\subsection{Other dopants}

Apart from polymers and acids, there are other types of dopants, such as additives, small molecule compounds, metal salts, etc., for the synthesis of hydrogels. They are used either to increase the conductivity and mechanical properties or achieve specific features of hydrogels, ${ }^{26,27,104-106,122}$ realizing the promising utilizations in e-skins.

Additive dopants (rGO, CNT, clays, etc.) are widely used to enhance both the mechanical property and conductivity of hydrogels. ${ }^{26,105,106}$ Chen et al. synthesized a poly(2-acrylamido-2methyl-1-propanesulfonic acid-co-acrylamide) hydrogel doped by rGO to increase the electronic conductivity of the system (Fig. 7a1 and a2). ${ }^{105}$ In their work, the hydrogel is further fabricated with a thermal-sensitive elastomer to form a coreshell segmental structure, having potential in flexible strain and temperature sensors and flexible electronic devices (Fig. 7a3). ${ }^{105}$ Similarly, CNT can also be doped into the hydrogel to endow conductivity. Roshanbinfar and co-authors reported a conductive biohybrid hydrogel by doping multiwall carbon nanotubes (MWCNT) into a pericardial matrix to generate PMCNThydrogel, which provides an appropriate environment for the pluripotent stem cell-derived cardiomyocytes growth. ${ }^{106}$ The conductivity and biocompatibility enable it to be a great candidate in e-skins. Interestingly, clays such as Laponite ${ }^{\circledR}$ may also be an excellent dopant with the conductive property. Tondera et al. prepared a conductive hydrogel with excellent stretchability and adhesivity, which can be used to fabricate

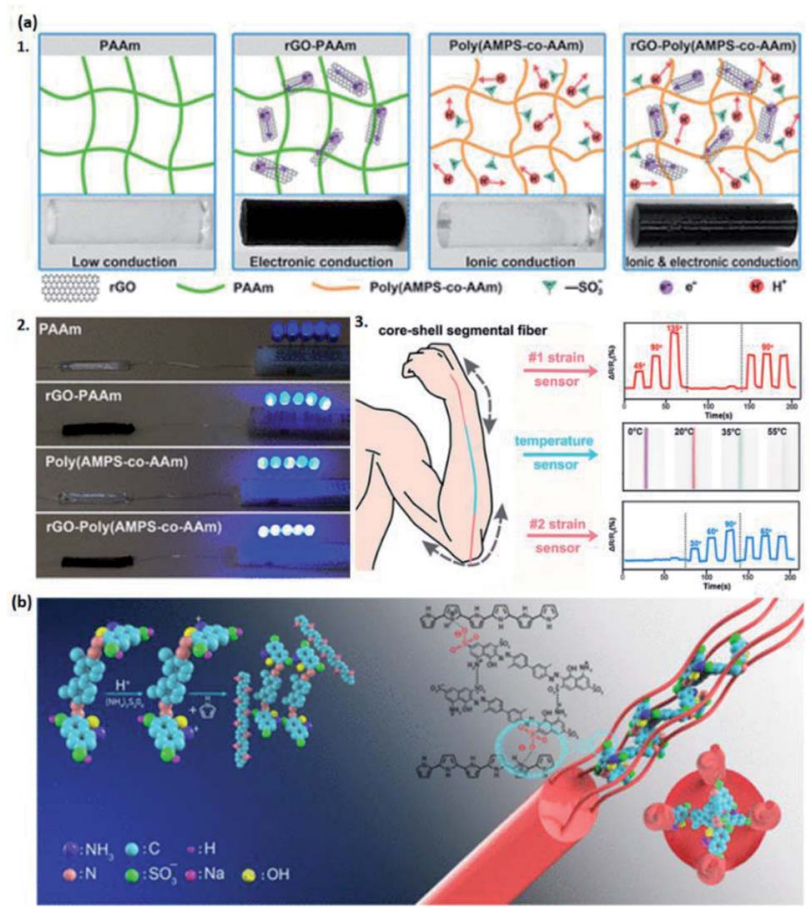

Fig. 7 (a) The illustration of rGO-poly(AMPS-CO-AAm) hydrogels with enhanced conductivity by (a1) designing ionic and electronic conductivities. (a2) The comparison of conductivity of the LED indicators for hydrogels containing different components. (a3) The potential applications of rGO-poly(AMPS-co-AAm) hydrogels in strain and temperature sensors. (Adapted with permission from ref. ${ }^{105}$. Copyright 2018, American Chemical Society). (b) Schematic representation of the PPy-based hydrogel doped by small molecule compounds TB. (Adapted with permission from ref. ${ }^{104}$. Copyright 2019, American Chemical Society). 
materials in tissue engineering. ${ }^{26}$ After the doping by Laponite ${ }^{\circledR}$, the poly(ethylene-3,4-diethoxy)-based hydrogel gained enhanced conductivity and mechanical properties. ${ }^{26}$ Such flexible, biocompatible hydrogels are a desirable material for fabricating e-skins.

Besides, small molecule components are also reported as dopants in preparing conductive hydrogels. ${ }^{27,104}$ For example, Yang and co-authors developed a PPy-based conductive hydrogel and crosslinked by trypan blue (TB) as a dopant (Fig. 7b). ${ }^{\mathbf{1 0 4}}$ By tuning the concentrations of $\mathrm{TB}$, different electrochemical properties can be achieved, and this hydrogel is flexible and exhibits high biocompatibility, ${ }^{\mathbf{1 0 4}}$ which is promising in designing e-skins or soft electronics. Also, Ding et al. reported a conductive hydrogel network by polymerizing PANI nanostructure in the presence of heparin as a dopant, which is crosslinked by methacrylate. ${ }^{27}$ It exhibits excellent conductivity and biocompatibility. Such hydrogels can be applied in biomaterials in tissue engineering, ${ }^{27}$ and are expected to be used in flexible electronics.

Another possible dopant for conductive hydrogels is metal salt, which promotes the ionic conductivity of the system. Recently, Yin et al. adopted a polypyrrole sodium alginate hydrogel doped with ferric chloride and sodium dodecylbenzene sulfonate, respectively. ${ }^{\mathbf{1 2 2}}$ Specifically, the former hydrogel exhibits better stability and electrochemical properties, but both of them are ideal materials to fabricate e-skins and flexible electronics. ${ }^{122}$

Several studies have demonstrated the diverse applications for these materials, which depend on the types of dopants and the functionalities the hydrogel provides. ${ }^{123,124}$ A typical case is the biosensor based on conductive hydrogels with superior flexibility and sensitivity, which are the most favored for either food detection such as food and water contaminant tests or healthcare monitoring to accurately assess the diagnosis of patients. ${ }^{\mathbf{1 2 5}}$ For instance, Ma et al. designed a wireless sensor for food spoilage detection, which was assembled by p-toluene sulfonate hexahydrate (PTS)-doped PANI hydrogels. ${ }^{\mathbf{1 2 4}}$ The material is highly sensitive to amines and meets the requirements for food detection since PTS is allowed to be used as a food additive based on the United States Food and Drug Administration (FDA) regulations. ${ }^{124}$ On the other hand, a nitrogen-doped graphene hydrogel reported by Chen and coauthors illustrated an effective solution in supercapacitors since it showed ultrafast discharge/charge rate and higher power density, indicating promising utilization in vehicle, lifts, or flexible robot systems. ${ }^{123}$

\section{Self-assembly}

Compared with polymerization, conductive hydrogels prepared by self-assembly can typically achieve weaker interactions such as p-p stacking, electrostatic interactions, van der Waals forces, hydrogen bonds, and hydrophobic interactions. ${ }^{126-137}$ Accordingly, better functionalities, mechanical properties, and interfacial affinity can be achieved for self-assembled hydrogels, improving the biocompatibility of synthetic polymers. ${ }^{38}$ Moreover, self-assembly plays a vital role in generating multifunctional conductive hydrogels in comparison with the copolymerization and doping strategies because no reactions are involved during this process. In this way, self-assembled conductive hydrogels have attracted widespread attention, and they are promising candidates in e-skins.

\section{$4.1 \pi-\pi$ stacking}

Induced by the strong interaction, $\pi-\pi$ stacking is one of the most important contributors for the self-assembly of conductive hydrogels. To endow these hydrogels with high conductivity, some great candidates could be graphene oxide (GO) or conductive molecules and polymers, and all of them contain conjugated $\mathrm{p}$ bonds, generating high strength for hydrogels. ${ }^{126-131}$ Such kinds of conductive hydrogels may potentially be used in flexible electronics and e-skins.

In general, the graphene-based component is selected as a raw material to synthesize conductive hydrogels due to its excellent conductivity and improved mechanical properties of stacking by the conjugated bonds. ${ }^{126,128,130,131}$ Introducing a onepot hydrothermal reaction is a typical way to form graphenebased conductive hydrogels through the self-assembly process. $^{126,128,130,131} \mathrm{Xu}$ and co-authors (2010) first demonstrated a facile one-step hydrothermal approach to construct a graphene-based hydrogel, featuring electrical conductivity, mechanical properties, thermal stability, and biocompatibility (Fig. 8a). ${ }^{131}$ Owing to the $\pi-\pi$ interaction as the main driving force, the two-dimensional GO can self-assemble into the 3D hydrogel network. ${ }^{131}$ The as-prepared hydrogel is expected to be applied in flexible electronics because of its biocompatibility and conductivity. Likewise, Sheng and co-authors fabricated

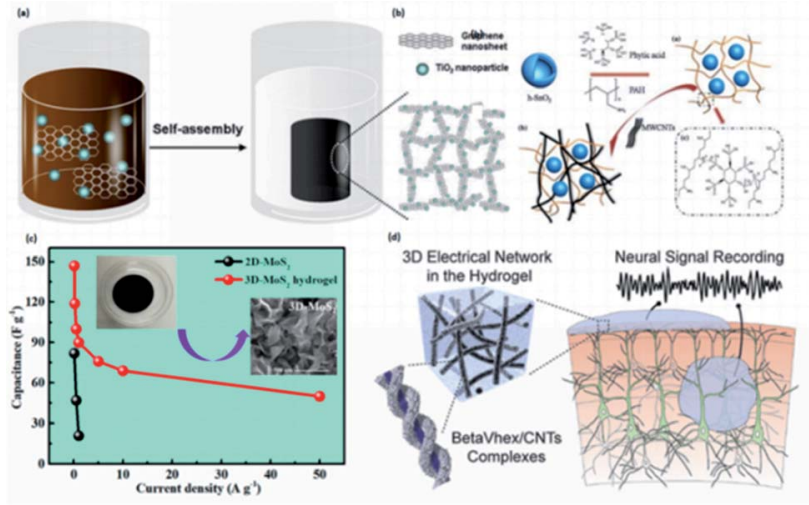

Fig. 8 The self-assembled conductive hydrogels are driven by four main types of secondary interactions. (a) Schematic illustration of graphene nanosheets driven by $\pi-\pi$ stacking through self-assembly. (Adapted with permission from ref. ${ }^{111}$ ). (Copyright 2013, American Chemical Society). (b) The mechanism of supramolecular selfassembly of PAH wrapped $\mathrm{SnO}_{2}$ anode and the addition of MWCNT to achieve electron transfer. (Adapted with permission from ref. ${ }^{133}$. Copyright 2016, Springer). (c) The illustration of $\mathrm{MoS}_{2}$-based hydrogel shows higher capacitance than 2D-MoS . (Adapted with permission from ref. ${ }^{137}$. Copyright 2019, American Chemical Society). (d) The representation of self-assembled betaVhex nanofibers and betaVhex/ CNTs complexes to form a hydrogel system and its utilization in neural signal monitoring. (Adapted with permission from ref. ${ }^{148}$. Copyright 2020, American Chemical Society). 
self-assembled chemical-reduced GO sheets via $\pi-\pi$ stacking with the promotion of sodium ascorbate, forming threedimensional graphene hydrogel by hydrothermal reaction, and it is an ideal material in supercapacitor manufacturing. ${ }^{\mathbf{1 3 0}}$ Moreover, graphene oxide can be incorporated with DNA chains to provide biocompatibility for the hydrogels so that they have potential in biochemical applications. Xu et al. (2010) successfully adopted a multifunctional hydrogel, having excellent mechanical strength, self-repairing ability, and can be used to prepare graphene-based biomaterials, ${ }^{126}$ flexible electronics devices, and e-skins. Furthermore, the DNA chains and GO nanosheets are driven by $\pi-\pi$ interactions through selfassembly, binding them together into a 3D network. ${ }^{\mathbf{1 2 6}}$ Besides, if GO nanosheets are combined with other particles, the mechanical properties of the hydrogel could be further enhanced. Zhang et al. developed a 3D nanocomposite conductive hydrogel based on self-assembled graphene nanosheets and $\mathrm{TiO}_{2}$ nanoparticles via a one-pot hydrothermal reaction. ${ }^{128}$ It is noteworthy that the $\pi-\pi$ stacking existing between GO nanosheets enables the formation of selfassembled hydrogels, which can be applied in fabricating energy storage devices. ${ }^{\mathbf{1 2 8}}$

Other materials such as conductive molecules and polymer blending may also be driven by the $\pi-\pi$ interaction. ${ }^{127,129}$ Components with low molecular weight combined with metal cations is typically used for supramolecular assembly of conductive hydrogels, ${ }^{138,139}$ and $\pi-\pi$ stacking as one major driving force for conjugated molecules, endowing excellent mechanical properties and self-healing capability. ${ }^{129} \mathrm{Hu}$ et al. developed a conductive hydrogel by incorporating adenosine $5^{\prime}$ monophosphate (AMP) and manganese ions $\left(\mathrm{Mn}^{2+}\right)$ through self-assembly to synthesize AMP-Mn hydrogel, followed by crosslinking with PVA. ${ }^{129}$ The self-assembly is due to the existence of $\pi-\pi$ stacking between nucleobases, and the asprepared hydrogel has wide applications in energy storage, ${ }^{129}$ enabling it to be a desirable candidate for e-skins. On the other hand, polymer blending is regarded as a viable source for fabricating host-guest self-assembly of conductive hydrogels. ${ }^{127}$ $\mathrm{Xu}$ et al. (2019) successfully synthesized a conductive hydrogel via host-guest self-assembly of poly- $\beta$-cyclodextrin $(\mathrm{P} \beta-\mathrm{CD})$ and PEDOT blended with adamantyl-modified sulfated alginate ( $\mathrm{S}$ Alg-Ad) through $\pi-\pi$ stacking interactions. ${ }^{127}$ Such hydrogels have high mechanical properties, self-healing ability, and injectability ${ }^{\mathbf{1 2 7}}$ and may be utilized in soft electronics or e-skins.

The studies about conductive hydrogels driven by $\pi-\pi$ stacking reveal the effective solutions of supercapacitors, endowing them with superior flexibility, stretchability, and high surface area for electrochemical reactions. ${ }^{\mathbf{1 2 0 , 1 4 0 , 1 4 1}} \mathrm{Xu}$ and coauthors have successfully developed a graphene/Ni(OH $)_{2}$ composite hydrogel, which was further assembled into a highperformance solid-state supercapacitor. ${ }^{\mathbf{1 4 0}}$ After that, $\mathrm{Xu}$ et al. reported another flexible supercapacitor fabricated by a 3D graphene hydrogel film, formed via $\pi-\pi$ stacking. ${ }^{\mathbf{1 4 1}}$ Such supercapacitors can achieve desirable gravimetric specific capacitance, cyclic stability, and excellent mechanical strength, indicating a bright future for flexible energy storage devices. ${ }^{\mathbf{1 4 1}}$ Besides the role as electronic skins for healthcare or biomedical applications, hydrogel-based flexible supercapacitors may also have wide utilization in electronic paper, energy driven devices, robotic systems, and roll-up displays, paving a path on designing smart electronics with more functionalities. ${ }^{\mathbf{1 4 2}}$

\subsection{Electrostatic interaction and van der Waals forces}

Electrostatic interactions and van der Waals forces are two types of noncovalent interactions with similar strengths between polymer chains. ${ }^{\mathbf{1 4 3}}$ However, van der Waals forces are generally the weak interactions between neutral molecules or polymers, while the electrostatic interaction involves the components with ionic groups. ${ }^{144}$ Both of them play an important role in driving the formation of conductive hydrogels. In the typical strategy, the selfassembled networks are incorporated with mechanically strong additives such as nanosheets or GOs to endow the hydrogels with robustness. Thus, they are the desirable materials in flexible electronic devices and biomaterial synthesis. ${ }^{\mathbf{1 3 2 - 1 3 4}}$

Electrostatic interactions are frequently employed to design conductive hydrogels via self-assembly, which refers to two groups of molecules with opposite charges attracted to each other, ${ }^{\mathbf{1 4 5}}$ forming a noncovalent interaction and contributing to the driven force to generate conductive hydrogels through selfassembly. Typically, the self-assembly process takes place between small natural molecules or polymer chains. ${ }^{132,133}$ For example, Patel et al. reported a hydrogel-based nanocomposite fibrous film fabricated by conductive graphene and two kinds of biocompatible polysaccharides (chitosan and gellan gum). ${ }^{\mathbf{1 3 2}}$ The existence of electrostatic interactions between positivelycharged chitosan and negatively-charged gellan gum contributes to the main driving force of self-assembly. ${ }^{\mathbf{1 3 2}}$ The addition of graphene enables excellent wettability, conductivity, and mechanical properties of the hydrogel, and it has great potential in biomaterial applications. ${ }^{\mathbf{1 3 2}}$ In their work, since the film has a unidirectional hierarchy, promoting the myoblasts to align correctly, and therefore, it is expected to design cell-instructive biomaterials. ${ }^{132}$ Also, Shi and co-authors mainly utilized the electrostatic interaction between the phytic acid (gelator) and poly(allylamine hydrochloride) (PAH) chains, followed by incorporation with $\mathrm{SnO}_{2}$, which is expected to have the excellent capacity by selfassembly process (Fig. 8b). ${ }^{133}$ Then, the prepared hydrogel is combined with MWCNT to improve the conductivity of the whole system. Specifically, there is a synergistic effect between PAH and MWCNTs and improving the conductivity and self-healing ability of the hydrogel is benefited from the existence of electrostatic effects and ionic interactions. ${ }^{133}$ Therefore, such hydrogels have a promising application in manufacturing anode for lithium-ion batteries, ${ }^{\mathbf{1 3 3}}$ which could be further designed to synthesize eskins or flexible electronics.

Introducing van der Waals force is also a promising strategy to synthesize conductive hydrogels through the self-assembly process. With the functionalization of specific groups on the materials, the self-assembly process can achieve different properties. For instance, Liu et al. developed a conductive hydrogel using a surface charge control strategy, and it is based on the self-assembly of three-dimensional molybdenum disulfide $\left(\mathrm{MoS}_{2}\right)$ nanosheets formed through van der Waals 
interaction (Fig. 8c). ${ }^{137}$ Unlike the traditional $\mathrm{MoS}_{2}$ material, this $\mathrm{MoS}_{2}$-based hydrogel exhibits higher capacitance, having great potential in fabricating supercapacitors, ${ }^{137}$ approaching a promising application in e-skins.

The existence of electrostatic interactions or van der Waals forces between polymer chains endows conductive hydrogels with excellent mechanical properties, indicating a promising future in flexible strain sensors. ${ }^{\mathbf{4 6 , 1 4 7}}$ Wang et al. synthesized a PVA hydrogel reinforced with zwitterionic PSBMA chains via electrostatic and dipole-dipole interactions. ${ }^{\mathbf{1 4 6}}$ The as-prepared hydrogels were utilized as a flexible strain sensor for pulse detection, showing superior accuracy and sensitivity to the subtle physiological signals. ${ }^{\mathbf{1 4 6}}$ Similarly, a flexible and wearable strain sensor was also developed by Xia et al., which is composed of polyacrylamide (PAAm)/chitosan (CS) hybrid hydrogels, and the conductive c-MWCNTs were fixed on the network through electrostatic interactions. ${ }^{147}$ The physiological signals such as bending, movement of the waist, neck, and elbows can also be monitored once the hydrogel-based strain sensor is attached to human skin. ${ }^{\mathbf{1 4 7}}$

\subsection{Self-assembly based on other stronger interactions}

Among all the self-assembly approaches through noncovalent interactions to prepare conductive hydrogels, hydrogen bonding and hydrophobic interactions are two types of forces, which are much stronger than others. Normally, the conductivity is achieved by incorporating conductive polymers or CNTs through self-assembly. Meanwhile, the mechanical properties of hydrogels can also be enhanced, enabling them to be applied in flexible electronics. ${ }^{36,134}$

Hydrogen bonding can be utilized to design conductive hydrogels via the self-assembly process, and due to its higher bond energy, the hydrogels are robust and have excellent properties. For example, Nam et al. reported a peptide-based conductive hydrogel with biocompatibility and biostability by self-assembling betaVhex and peptide via hydrogen bond interactions to synthesize betaVhex, followed by incorporating conductive CNT (Fig. 8d). ${ }^{\mathbf{1 4 8}}$ Then, the prepared betaVhex/CNTs hydrogel is used to form a soft neural interface, which can magnify the signals and prevent the signal from being dissipated. ${ }^{\mathbf{4} 8}$ Such materials can be further applied to make e-skins.

Similarly, hydrophobic interaction is also used to drive the formation of the hydrogel by self-assembly. In fact, the hydrophobic interactions are generally stronger than that of hydrogen bonds, leading to relatively high mechanical properties and fast self-repairing ability of hydrogels, ${ }^{135}$ and the self-assembly performance depend on plenty of factors such as temperature, hydrophobic functional groups, and the shape of materials. ${ }^{149}$ In most cases, the hydrophobic interaction takes place between nanoparticles, driving the formation of self-assembled networks. ${ }^{\mathbf{1 3 5 , 1 3 6}}$ $\mathrm{Xu}$ et al. developed an electroconductive hydrogel based on selfassembling PEDOT:PSS and PEG-peptide together, which is mainly driven by hydrophobic interactions between PEDOT nanoparticles, and p-p stacking among PSS and PEDOT, attributing to the self-healing ability of the hydrogel, and it can be applied in adopting cell cultures. ${ }^{\mathbf{1 3 5}}$ Recently, Gao and co-authors successfully developed a DNA-based hydrogel, which was incorporated by conductive CNTs and AuNPs nanoparticles through self-assembly. ${ }^{136}$ In their work, the DNA chains are wrapped around the CNT walls via strong hydrophobic interactions, promoting the assembly of the hydrogel, which exhibits excellent conductivity and mechanical properties, approaching a more desirable application in flexible electronic devices. ${ }^{\mathbf{1 3 6}}$

Hydrogen bond and hydrophobic powered conductive hydrogels demonstrate a strong impact in the applications of electronic skins, especially for flexible strain sensors for monitoring devices in the biomedical field. ${ }^{\mathbf{1 5 0 , 1 5 1}}$ Liu et al. prepared a strain sensor based on GelMA-TA-carbon hydrogel, which was driven by hydrogen bonding with superior mechanical properties, flexibility, and adhesiveness. ${ }^{\mathbf{1 5 0}}$ The as-prepared material was further demonstrated as a wound-healing material, indicating a new innovation in the biomedical area. ${ }^{\mathbf{1 5 0}}$ Besides, Ye and co-authors reported a multi-functional organohydrogel sensor for human motion detection under either large or subtle changes of pressure. ${ }^{\mathbf{1 5 1}}$ Notably, upon both compressive and tensile deformation, the sensor can maintain its sensitivity, stability, and durability, ${ }^{151}$ suggesting extensive utilization in healthcare, injectable materials, or seawater desalination.

\section{Perspective and discussion}

Conducive hydrogel is of utmost importance for e-skins by virtue of its flexibility, stretchability, self-healing properties, sensitivity, and adhesiveness, ${ }^{66-73,86,152}$ which have made it a promising alternative for traditional inorganic conductive materials. ${ }^{153}$ Thus, its fabrication strategies continue to be a great impetus to achieve incremental performances of conductive hydrogels.

As one of the most popular approaches, copolymerization can not only control the crosslink density and ion contents in the hydrogel network by directly tuning the ratios among different monomers, ${ }^{66}$ but is also useful in modifying the special functional groups on the hydrogel backbones and forming covalent crosslinking and physical interactions among chains through grafting. ${ }^{72,74}$ However, it is time-consuming and requires strict controls for preparation, ${ }^{154}$ and lacks mechanical and conductive stability when conductive hydrogels are prepared..$^{23}$

Compared to copolymerization, doping can also be employed to modify the mechanical and conductive properties by controlling the amount of dopants. ${ }^{107}$ But what really matters is, based on this chemical approach, the functions of the hydrogels are much easier to modulate with high accuracy, and there is a possibility for dopants to exhibit reversible properties in the hydrogel structures, endowing high sensitivity and multifunctional responsiveness for the hydrogels. ${ }^{37}$ Moreover, the performance of self-assembled conductive hydrogels can be further enhanced using dopants, which help to tailor the morphology of the self-assembled hydrogels due to the unique functional groups in dopants, ${ }^{37}$ forming conductive hydrogels for various functions. Hence, doping can be regarded as a complementary strategy for copolymerization and selfassembly in fabricating conductive hydrogels, raising the prospects in the applications of flexible electronics. 


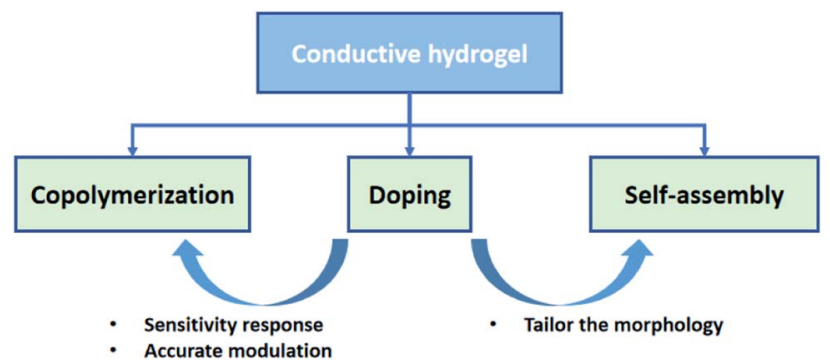

Fig. 9 The relationship between different fabrication approaches for conductive hydrogels.

As mentioned above, self-assembly is also a critical approach to fabricate supramolecular hydrogels, endowing the hydrogels with self-healing ability, which plays a significant role for electronic skins. ${ }^{129}$ Owing to the various categories of driving forces, such as p-p stacking, ${ }^{126-131}$ hydrophobic interaction, ${ }^{36,135,136}$ van der Waals forces, ${ }^{137}$ hydrogen bonds ${ }^{134}$ and electrostatic interactions, ${ }^{132,133}$ self-assembly can be adopted into different kinds of hydrogel synthesis, conferring a superior degree of selfhealing capacity in the materials.

Based on these three fundamental strategies for the rational design of conductive polymers, particularly for hydrogels, we should pour much more attention on developing combined strategies such as polymerization-doping or self-assembly-doping, realizing superior mechanical strength conductivity and functionality simultaneously via different optimization mechanisms (Fig. 9).

In summary, this review discusses the recent advances of conductive hydrogels and proposes a theoretical framework to synthesize conductive hydrogels, which can be mainly classified into copolymerization, doping, and self-assembly, and their advantages and disadvantages are also discussed critically. Copolymerization and self-assembly methods can generate tunable and self-healable conductive hydrogels, respectively, while doping can usually be utilized to improve the stability and functionalities of hydrogels. Furthermore, the potential applications for conductive hydrogels in e-skins are also highlighted.

In the future, further efforts are still required to figure out appropriate combinations of possible strategies in terms of copolymerization, doping, and self-assembly. These chemical methodologies can be applied to design a new class of chemical tools in fabricating conductive hydrogels so its performance in the utilization of e-skins and flexible electronic devices can be further optimized. Thus, it is expected that in the future, these three approaches will still dominate in synthesizing conductive hydrogels with improved mechanical, conductive properties and multifunctionalities, providing a breakthrough in the usages of e-skins.

\section{Author contributions}

C. J. Li conceived the whole manuscript and organized the materials. The author has approved the final version of the manuscript.

\section{Conflicts of interest}

There are no conflicts to declare.

\section{Acknowledgements}

We would like to acknowledge Dr Han Zhang and Dr Dimitrios Papageorgiou at Queen Mary University of London for their patient and professional guidance about hydrogels, which provides the inspiration for the review. The author also thanks Miss Jingyi Shi, Ziyang Li, Peishan Li, Zekun Liu, Xinyu Zhao for their support and suggestions on the coursework. We gratefully acknowledge the constructive suggestions provided by Dr. Wei HUANG from the Chinese University of Hong Kong.

\section{Notes and references}

1 Z. Wang, Y. Cong and J. Fu, J. Mater. Chem. B, 2020, 8, 34373459.

2 T.-H. Han, H. Kim, S.-J. Kwon and T.-W. Lee, Mater. Sci. Eng., $R$, 2017, 118, 1-43.

3 Z. Zhang, Z. Chen, Y. Wang and Y. Zhao, Proc. Natl. Acad. Sci. U. S. A., 2020, 117, 18310.

4 S. Zhang, S. Li, Z. Xia and K. Cai, J. Mater. Chem. B, 2020, 8, 852-862.

5 S. R. Forrest, Nature, 2004, 428, 911-918.

6 Z. Wang, Y. Cong and J. Fu, J. Mater. Chem. B, 2020, 8, 34373459.

7 D.-H. Kim, J.-H. Ahn, W. M. Choi, H.-S. Kim, T.-H. Kim, J. Song, Y. Y. Huang, Z. Liu, C. Lu and J. A. Rogers, Science, 2008, 320, 507.

8 G.-T. Hwang, D. Im, S. E. Lee, J. Lee, M. Koo, S. Y. Park, S. Kim, K. Yang, S. J. Kim, K. Lee and K. J. Lee, ACS Nano, 2013, 7, 4545-4553.

9 K. Liu, S. Wei, L. Song, H. Liu and T. Wang, J. Agric. Food Chem., 2020, 68, 7269-7280.

10 X. Yang, X. Zhang, Q. Guan and X. Zhang, J. Mater. Chem. C, 2021, 9, 2815-2822.

11 J. Zhang, Z. Lei, S. Luo, Y. Jin, L. Qiu and W. Zhang, ACS Appl. Nano Mater., 2020, 3, 4845-4850.

12 X. Liu, Y.-Z. Long, L. Liao, X. Duan and Z. Fan, ACS Nano, 2012, 6, 1888-1900.

13 W. K. Chee, H. N. Lim, Z. Zainal, N. M. Huang, I. Harrison and Y. Andou, J. Phys. Chem. C, 2016, 120, 4153-4172.

14 H.-P. Phan, Y. Zhong, T.-K. Nguyen, Y. Park, T. Dinh, E. Song, R. K. Vadivelu, M. K. Masud, J. Li, M. J. A. Shiddiky, D. Dao, Y. Yamauchi, J. A. Rogers and N.-T. Nguyen, ACS Nano, 2019, 13, 11572-11581.

15 D. Shahrjerdi and S. W. Bedell, Nano Lett., 2013, 13, 315320.

16 K.-J. Moon, T.-I. Lee, J.-H. Choi, J. Jeon, Y. H. Kang, J. P. Kar, J. H. Kang, I. Yun and J.-M. Myoung, ACS Nano, 2011, 5, 159-164.

17 X. Liu, Y.-Z. Long, L. Liao, X. Duan and Z. Fan, ACS Nano, 2012, 6, 1888-1900.

18 J. P. Rojas, G. A. Torres Sevilla, M. T. Ghoneim, S. B. Inayat, S. M. Ahmed, A. M. Hussain and M. M. Hussain, ACS Nano, 2014, 8, 1468-1474.

19 K. Teng, Q. An, Y. Chen, Y. Zhang and Y. Zhao, ACS Biomater. Sci. Eng., 2021, 7, 1302-1337. 
20 Y. Lee, J. Park, A. Choe, S. Cho, J. Kim and H. Ko, Adv. Funct. Mater., 2020, 30, 1904523.

21 Y. Cao, Y. J. Tan, S. Li, W. W. Lee, H. Guo, Y. Cai, C. Wang and B. Tee, Nat. Electron., 2019, 2, 75.

22 J. Stejskal, Chem. Pap., 2017, 71, 269-291.

23 F. Fu, J. Wang, H. Zeng and J. Yu, ACS Mater. Lett., 2020, 2, 1287-1301.

24 T.-H. Le, Y. Kim and H. Yoon, Polymers, 2017, 9(4), 150.

25 K. Liu, S. Wei, L. Song, H. Liu and T. Wang, J. Agric. Food Chem., 2020, 68, 7269-7280.

26 C. Tondera, T. F. Akbar, A. K. Thomas, W. Lin, C. Werner, V. Busskamp, Y. Zhang and I. R. Minev, Small, 2019, 15, 1901406.

27 H. Ding, M. Zhong, Y. J. Kim, P. Pholpabu, A. Balasubramanian, C. M. Hui, H. He, H. Yang, K. Matyjaszewski and C. J. Bettinger, ACS Nano, 2014, 8, 4348-4357.

28 Y. Zhao, C. Shi, X. Yang, B. Shen, Y. Sun, Y. Chen, X. Xu, H. Sun, K. Yu, B. Yang and Q. Lin, ACS Nano, 2016, 10, 5856-5863.

29 M. Choi, J. W. Choi, S. Kim, S. Nizamoglu, S. K. Hahn and S. H. Yun, Nat. Photonics, 2013, 7, 987-994.

30 I. Jeon, J. Cui, W. R. K. Illeperuma, J. Aizenberg and J. J. Vlassak, Adv. Mater., 2016, 28, 4678-4683.

31 P. Chakraborty, T. Guterman, N. Adadi, M. Yadid, T. Brosh, L. Adler-Abramovich, T. Dvir and E. Gazit, ACS Nano, 2019, 13, 163-175.

32 Y.-J. Liu, W. Cao, M.-G. Ma and P. Wan, ACS Appl. Mater. Interfaces, 2017, 9 (30), 25559-25570.

33 Z. Wang, H. Zhou, W. Chen, Q. Li, B. Yan, X. Jin, A. Ma, H. Liu and W. Zhao, ACS Appl. Mater. Interfaces, 2018, 10, 14045-14054.

34 S. Mandal, A. Seth, V. Yadav, S. Kumari, M. Kumar and U. Ojha, ACS Appl. Polym. Mater., 2020, 2, 618-625.

35 Y. Liang, X. Zhao, T. Hu, B. Chen, Z. Yin, P. X. Ma and B. Guo, Small, 2019, 15, 1900046.

36 J. Nam, H.-K. Lim, N. H. Kim, J. K. Park, E. S. Kang, Y.-T. Kim, C. Heo, O.-S. Lee, S.-G. Kim, W. S. Yun, M. Suh and Y. H. Kim, ACS Nano, 2020, 14, 664-675.

37 Z. Ma, W. Shi, K. Yan, L. Pan and G. Yu, Chem. Sci., 2019, 10, 6232-6244.

38 M. Halperin-Sternfeld, M. Ghosh and L. Adler-Abramovich, in Supramolecular Chemistry of Biomimetic Systems, ed. J. Li, Springer Singapore, Singapore, 2017, pp. 9-35.

39 M. W. Handlogten, J. Wang and S. Ahuja, Biotechnol. Bioeng., 2020, 117, 1329-1336.

40 F. Alam and K. Balani, Adhesion force of staphylococcus aureus on various biomaterial surfaces, J. Mech. Behav. Biomed. Mater., 2017, 65, 872-880.

41 F. Alam and K. Balani, J. Mech. Behav. Biomed. Mater., 2017, 65, 872-880.

42 Y. Yu, H. Yuk, G. A. Parada, Y. Wu, X. Liu, C. S. Nabzdyk, K. Youcef-Toumi, J. Zang and X. Zhao, Adv. Mater., 2019, 31, 1807101.

43 I. Jeon, J. Cui, W. R. K. Illeperuma, J. Aizenberg and J. J. Vlassak, Adv. Mater., 2016, 28, 4678-4683.

44 F. Zhu, J. Lin, Z. L. Wu, S. Qu, J. Yin, J. Qian and Q. Zheng, ACS Appl. Mater. Interfaces, 2018, 10, 13685-13692.
45 Q. Hu, G. Li, X. Liu, B. Zhu, X. Chai, Q. Zhang, J. Liu and C. He, Angew. Chem., Int. Ed., 2019, 58, 4318-4322.

46 L. Zhou, L. Fan, X. Yi, Z. Zhou, C. Liu, R. Fu, C. Dai, Z. Wang, X. Chen, P. Yu, D. Chen, G. Tan, Q. Wang and C. Ning, ACS Nano, 2018, 12, 10957-10967.

47 L. Han, L. Yan, M. Wang, K. Wang, L. Fang, J. Zhou, J. Fang, F. Ren and X. Lu, Chem. Mater., 2018, 30, 5561-5572.

48 A. R. Spencer, A. Primbetova, A. N. Koppes, R. A. Koppes, H. Fenniri and N. Annabi, ACS Biomater. Sci. Eng., 2018, 4, 1558-1567.

49 B. Liu, P. Soares, C. Checkles, Y. Zhao and G. Yu, Nano Lett., 2013, 13, 3414-3419.

50 F. Wang, J. Tseng, Z. Liu, P. Zhang, G. Wang, G. Chen, W. Wu, M. Yu, Y. Wu and X. Feng, Adv. Mater., 2020, 32, 2000287.

51 Z. Wang, H. Zhou, J. Lai, B. Yan, H. Liu, X. Jin, A. Ma, G. Zhang, W. Zhao and W. Chen, J. Mater. Chem. C, 2018, 6, 9200-9207.

52 V. P. Chuang, J. Y. Cheng, T. A. Savas and C. A. Ross, Nano Lett., 2006, 6, 2332-2337.

53 L. He, Q. Xiao, Y. Zhao, J. Li, S. Reddy, X. Shi, X. Su, K. Chiu and S. Ramakrishna, ACS Appl. Mater. Interfaces, 2020, 12, 53150-53163.

54 Y. Hu, P. Shen, N. Zeng, L. Wang, D. Yan, L. Cui, K. Yang and C. Zhai, ACS Appl. Mater. Interfaces, 2020, 12, 4228542293.

55 Y. Xu, M. Cui, P. A. Patsis, M. Günther, X. Yang, K. Eckert and Y. Zhang, ACS Appl. Mater. Interfaces, 2019, 11, 77157724.

56 Y. Xu, X. Yang, A. K. Thomas, P. A. Patsis, T. Kurth, M. Kräter, K. Eckert, M. Bornhäuser and Y. Zhang, ACS Appl. Mater. Interfaces, 2018, 10, 14418-14425.

57 M. R. Kesama, S. R. Dugasani, Y. J. Cha, J. Son, B. Gnapareddy, S. Yoo, D. K. Yoon and S. H. Park, Nanotechnology, 2019, 30, 245704.

58 J. Nam, H.-K. Lim, N. Kim, J. Park, E. Kang, Y. Kim, C. Heo, O.-S. Lee, S.-G. Kim, W. Yun, M. Suh and Y. Kim, ACS Nano, 2020, 14, 664-675.

59 Encyclopedia of Cancer, ed. M. Schwab, Springer Berlin Heidelberg, Berlin, Heidelberg, 2009, pp. 958-958.

60 A. Patel, Y. Xue, R. Hartley, V. Sant, J. R. Eles, X. T. Cui, D. B. Stolz and S. Sant, Biotechnol. Bioeng., 2018, 115, 2654-2667.

61 F. Poncin and G. Legeay, J. Biomater. Sci., Polym. Ed., 2003, 14, 1005-1028.

62 K. G. Neoh, R. Wang and E. T. Kang, in Biomaterials and Medical Device - Associated Infections, ed. L. Barnes and I. R. Cooper, Woodhead Publishing, Oxford, 2015, pp. 133161.

63 X. Peng, F. Zhao, Y. Peng, J. Li and Q. Zeng, Soft Matter, 2020, 16, 54-63.

64 R. Balint, N. J. Cassidy and S. H. Cartmell, Acta Biomater., 2014, 10, 2341-2353.

65 F. Fu, J. Wang, H. Zeng and J. Yu, ACS Mater. Lett., 2020, 2, 1287-1301.

66 L. Jiang, C. Gentile, A. Lauto, C. Cui, Y. Song, T. Romeo, S. M. Silva, O. Tang, P. Sharma, G. Figtree, J. J. Gooding 
and D. Mawad, ACS Appl. Mater. Interfaces, 2017, 9, 4412444133.

67 Z. Wang, J. Chen, Y. Cong, H. Zhang, T. Xu, L. Nie and J. Fu, Chem. Mater., 2018, 30, 8062-8069.

68 L. Wang, G. Gao, Y. Zhou, T. Xu, J. Chen, R. Wang, R. Zhang and J. Fu, ACS Appl. Mater. Interfaces, 2019, 11, 3506-3515.

69 S. Das, P. Martin, G. Vasilyev, R. Nandi, N. Amdursky and E. Zussman, Macromolecules, 2020, 53, 11130-11141.

70 S. Liu, O. Oderinde, I. Hussain, F. Yao and G. Fu, Polymer, 2018, 144, 111-120.

71 Y. Tan, Y. Zhang, Y. Zhang, J. Zheng, H. Wu, Y. Chen, S. Xu, J. Yang, C. Liu and Y. Zhang, Chem. Mater., 2020, 32, 76707678.

72 X. Li, X. Huang, H. Mutlu, S. Malik and P. Theato, Soft Matter, 2020, 16, 10969-10976.

73 Y. Long, Y. Chen, Y. Liu, G. Chen, W. Guo, X. Kang, X. Pu, W. Hu and Z. L. Wang, Nanoscale, 2020, 12, 12753-12759.

74 D. Chen, X. Zhao, X. Wei, J. Zhang, D. Wang, H. Lu and P. Jia, ACS Appl. Mater. Interfaces, 2020, 12, 53247-53256.

75 H. Dai, Y. Zhang, L. Ma, H. Zhang and H. Huang, Carbohydr. Polym., 2019, 215, 366-376.

76 X. Zhao, B. Guo and P. X. Ma, J. Mater. Chem. B, 2015, 3, 8459-8468.

77 R. Dong, X. Zhao, B. Guo and P. X. Ma, ACS Appl. Mater. Interfaces, 2016, 8, 17138-17150.

78 E. Jafarigol, M. B. Salehi and H. R. Mortaheb, Chem. Eng. Res. Des., 2020, 162, 74-84.

79 Y. Liang, B. Chen, M. Li, J. He, Z. Yin and B. Guo, Biomacromolecules, 2020, 21, 1841-1852.

80 Mandeep, A. Gulati and R. Kakkar, Chem. Eng. Res. Des., 2020, 153, 21-36.

81 S. Wang, J. Lei, X. Yi, L. Yuan, L. Ge, D. Li and C. Mu, ACS Appl. Polym. Mater., 2020, 2, 3016-3023.

82 C. Cui, Q. Fu, L. Meng, S. Hao, R. Dai and J. Yang, ACS Appl. Bio Mater., 2021, 4, 85-121.

83 X. Han, G. Xiao, Y. Wang, X. Chen, G. Duan, Y. Wu, X. Gong and H. Wang, J. Mater. Chem. A, 2020, 8, 23059-23095.

84 X.-W. Liu, Y.-X. Huang, X.-F. Sun, G.-P. Sheng, F. Zhao, S.-G. Wang and H.-Q. Yu, ACS Appl. Mater. Interfaces, 2014, 6, 8158-8164.

85 A. A. Adewunmi, S. Ismail and A. S. Sultan, J. Inorg. Organomet. Polym. Mater., 2016, 26, 717-737.

86 K. Balasubramanian and M. Burghard, J. Mater. Chem., 2008, 18, 3071-3083.

87 Z. Zhang, Z. Gao, Y. Wang, L. Guo, C. Yin, X. Zhang, J. Hao, G. Zhang and L. Chen, Macromolecules, 2019, 52, 25312541.

88 B. Lv, X. Bu, Y. Da, P. Duan, H. Wang, J. Ren, B. Lyu, D. Gao and J. Ma, Polymer, 2020, 210, 123021.

89 O. Zhanadilov, A. Mentbayeva, Z. Beisbayeva, M. Amze and Z. Bakenov, Mater. Today: Proc., 2021, DOI: 10.1016/ j.matpr.2020.11.989.

90 C. Yang, Z. Liu, C. Chen, K. Shi, L. Zhang, X.-J. Ju, W. Wang, R. Xie and L.-Y. Chu, ACS Appl. Mater. Interfaces, 2017, 9, 15758-15767.

91 Y. Shi, L. J. Pan, B. Liu, Y. Wang, Y. Cui, Z. Bao and G. Yu, J. Mater. Chem. A, 2014, 2, 6086-6091.
92 X. Liu, Q. Zhang and G. Gao, Chem. Eng. J., 2020, 394, 124898.

93 L. Tang, S. Wu, J. Qu, L. Gong and J. Tang, Materials, 2020, 13(18), 3947.

$94 \mathrm{H}$. Zhang, M. Yue, T. Wang, J. Wang, X. Wu and S. Yang, New J. Chem., 2021, 45, 4647-4657.

95 W. Li, F. Gao, X. Wang, N. Zhang and M. Ma, Angew. Chem., Int. Ed., 2016, 55, 9196-9201.

96 S. Banerjee, B. De, P. Sinha, J. Cherusseri and K. K. Kar, in Handbook of Nanocomposite Supercapacitor Materials I: Characteristics, ed. K. K. Kar, Springer International Publishing, Cham, 2020, pp. 341-350.

97 L.-Y. Hsiao, L. Jing, K. Li, H. Yang, Y. Li and P.-Y. Chen, Carbon, 2020, 161, 784-793.

98 J. Yang, C.-R. Han, J.-F. Duan, F. Xu and R.-C. Sun, Nanoscale, 2013, 5, 10858-10863.

99 Y. Wu, B. Guo and P. X. Ma, ACS Macro Lett., 2014, 3, 11451150.

100 D. Chen, X. Zhao, X. Wei, J. Zhang, D. Wang, H. Lu and P. Jia, ACS Appl. Mater. Interfaces, 2020, 12, 53247-53256.

101 Y. Huang, M. Zhong, F. Shi, X. Liu, Z. Tang, Y. Wang, Y. Huang, H. Hou, X. Xie and C. Zhi, Angew. Chem., Int. Ed., 2017, 56, 9141-9145.

102 H. Zhou, M. Wang, X. Jin, H. Liu, J. Lai, H. Du, W. Chen and A. Ma, ACS Appl. Mater. Interfaces, 2021, 13, 1441-1451.

103 A. Katzenberg, C. Muñoz Davila, B. Chen, T. Siboonruang and M. A. Modestino, ACS Appl. Polym. Mater., 2020, 2, 2046-2054.

104 C. Yang, P. Zhang, A. Nautiyal, S. Li, N. Liu, J. Yin, K. Deng and X. Zhang, ACS Appl. Mater. Interfaces, 2019, 11, 42584267.

105 J. Chen, H. Wen, G. Zhang, F. Lei, Q. Feng, Y. Liu, X. Cao and H. Dong, ACS Appl. Mater. Interfaces, 2020, 12, 75657574.

106 K. Roshanbinfar, Z. Mohammadi, A. Sheikh-Mahdi Mesgar, M. M. Dehghan, O. P. Oommen, J. Hilborn and F. B. Engel, Biomater. Sci., 2019, 7, 3906-3917.

107 L. Zhao, X. Li, Y. Li, X. Wang, W. Yang and J. Ren, Biomacromolecules, 2021, 22(3), 1273-1281.

108 S. Das, P. Chakraborty, S. Mondal, A. Shit and A. K. Nandi, ACS Appl. Mater. Interfaces, 2016, 8, 28055-28067.

109 Q. Wu, J. Wei, B. Xu, X. Liu, H. Wang, W. Wang, Q. Wang and W. Liu, Sci. Rep., 2017, 7, 41566.

110 Y. Bu, H.-X. Xu, X. Li, W.-J. Xu, Y. Yin, H. Dai, X. Wang, Z.-J. Huang and P.-H. Xu, RSC Adv., 2018, 8, 10806-10817.

111 L. Han, L. Yan, M. Wang, K. Wang, L. Fang, J. Zhou, J. Fang, F. Ren and X. Lu, Chem. Mater., 2018, 30(16), 5561-5572.

112 J. Chen, Q. Peng, T. Thundat and H. Zeng, Chem. Mater., 2019, 31(12), 4553-4563.

113 H. Ejima, J. Richardson, K. Liang, J. Best, M. G. van KoeverdenSuch, J. Cui and F. Caruso, Science, 2013, 341, 154-157.

114 S. Quideau, D. Deffieux and C. Douat-Casassus, Angew. Chem., 2011, 50, 586-621.

115 M. Shin, H.-A. Lee, M. Lee, Y. Shin, J.-J. Song, S.-W. Kang, D.-H. Nam, E. J. Jeon, M. Cho, M. Do, S. Park, M. S. Lee, 
J.-H. Jang, S.-W. Cho, K.-S. Kim and H. Lee, Nat. Biomed. Eng., 2018, 2, 304-317.

116 F. Behboodi-Sadabad, H. Zhang, V. Trouillet, A. Welle, N. Plumeré and P. A. Levkin, Adv. Funct. Mater., 2017, 27, 1700127.

117 S. Zhang, Y. Chen, H. Liu, Z. Wang, H. Ling, C. Wang, J. Ni, B. Çelebi-Saltik, X. Wang, X. Meng, H.-J. Kim, A. Baidya, S. Ahadian, N. Ashammakhi, M. R. Dokmeci, J. TravasSejdic and A. Khademhosseini, Adv. Mater., 2020, 32, 1904752.

118 L. Pan, A. Chortos, G. Yu, Y. Wang, S. Isaacson, R. Allen, Y. Shi, R. Dauskardt and Z. Bao, Nat. Commun., 2014, 5, 3002 .

119 Y. Shi, J. Zhang, A. M. Bruck, Y. Zhang, J. Li, E. A. Stach, K. J. Takeuchi, A. C. Marschilok, E. S. Takeuchi and G. Yu, Adv. Mater., 2017, 29, 1603922.

120 W. Zhang, P. Feng, J. Chen, Z. Sun and B. Zhao, Prog. Polym. Sci., 2019, 88, 220-240.

121 D. C. Bock, K. C. Kirshenbaum, J. Wang, W. Zhang, F. Wang, J. Wang, A. C. Marschilok, K. J. Takeuchi and E. S. Takeuchi, ACS Appl. Mater. Interfaces, 2015, 7, 13457-13466.

122 J. Yin, Q. Liu, J. Zhou, L. Zhang, Q. Zhang, R. Rao, S. Liu and T. Jiao, RSC Adv., 2020, 10, 10546-10551.

123 P. Chen, J.-J. Yang, S.-S. Li, Z. Wang, T.-Y. Xiao, Y.-H. Qian and S.-H. Yu, Nano Energy, 2013, 2, 249-256.

124 Z. Ma, P. Chen, W. Cheng, K. Yan, L. J. Pan, Y. Shi and G. Yu, Nano Lett., 2018, 18(7), 4570-4575.

125 A. Haleem, M. Javaid, R. P. Singh, R. Suman and S. Rab, Sensors International, 2021, 2, 100100.

126 Y. Xu, Q. Wu, Y. Sun, H. Bai and G. Shi, ACS Nano, 2010, 4, 7358-7362.

127 Y. Xu, M. Cui, P. A. Patsis, M. Günther, X. Yang, K. Eckert and Y. Zhang, ACS Appl. Mater. Interfaces, 2019, 11, 77157724.

128 Z. Zhang, F. Xiao, Y. Guo, S. Wang and Y. Liu, ACS Appl. Mater. Interfaces, 2013, 5, 2227-2233.

129 Y. Hu, P. Shen, N. Zeng, L. Wang, D. Yan, L. Cui, K. Yang and C. Zhai, ACS Appl. Mater. Interfaces, 2020, 12, 4228542293.

130 K. Sheng, Y. Xu, C. Li and G. Shi, New Carbon Mater., 2011, 26, 9-15.

131 Y. Xu, K. Sheng, C. Li and G. Shi, ACS Nano, 2010, 4, 43244330.

132 A. Patel, Y. Xue, R. Hartley, V. Sant, J. R. Eles, X. T. Cui, D. B. Stolz and S. Sant, Biotechnol. Bioeng., 2018, 115, 2654-2667.

133 Y. Shi, D. Ma, W. Wang, L. Zhang and X. Xu, J. Mater. Sci., 2017, 52, 3545-3555.
134 J. Nam, H.-K. Lim, N. H. Kim, J. K. Park, E. S. Kang, Y.-T. Kim, C. Heo, O.-S. Lee, S.-G. Kim, W. S. Yun, M. Suh and Y. H. Kim, ACS Nano, 2020, 14, 664-675.

135 Y. Xu, X. Yang, A. K. Thomas, P. A. Patsis, T. Kurth, M. Kräter, K. Eckert, M. Bornhäuser and Y. Zhang, ACS Appl. Mater. Interfaces, 2018, 10, 14418-14425.

136 M. Gao, A. Krissanaprasit, A. Miles, L. C. Hsiao and T. H. LaBean, J. Appl. Sci., 2021, 11.

137 C. Liu, X. Zhao, S. Wang, Y. Zhang, W. Ge, J. Li, J. Cao, J. Tao and X. Yang, ACS Appl. Energy Mater., 2019, 2, 4458-4463.

138 M.-O. M. Piepenbrock, G. Lloyd, N. Clarke and J. W. Steed, Chem. Rev., 2010, 110(4), 1960-2004.

139 M. Krogsgaard, V. Nue and H. Birkedal, Chemistry, 2016, 22, 844-857.

140 Y. Xu, X. Huang, Z. Lin, X. Zhong, Y. Huang and X. Duan, Nano Res., 2013, 6, 65-76.

141 Yuxi Xu, Xiaoqing Huang, Zhaoyang Lin, Xing Zhong, Yu Huang and Xiangfeng Duan, Nano Res., 2013, 6, 65-76.

142 X. Liang, G. Long, C. Fu, M. Pang, Y. Xi, J. Li, W. Han, G. Wei and Y. Ji, Chem. Eng. J., 2018, 345, 186-195.

143 B. Shi, Z. Wang and H. Wen, J. Mol. Liq., 2017, 241, 486-488. 144 T. Tsuchiya, in Encyclopedia of Geochemistry: A Comprehensive Reference Source on the Chemistry of the Earth, ed. W. M. White, Springer International Publishing, Cham, 2018, pp. 1473-1474.

145 Encyclopedia of Cancer, ed. M. Schwab, Springer Berlin Heidelberg, Berlin, Heidelberg, 2009, pp. 958-958.

146 Z. Wang, J. Chen, L. Wang, G. Gao, Y. Zhou, R. Wang, T. Xu, J. Yin and J. Fu, J. Mater. Chem. B, 2019, 7, 24-29.

147 S. Xia, S. Song, F. Jia and G. Gao, J. Mater. Chem. B, 2019, 7, 4638-4648.

148 J. Nam, H.-K. Lim, N. H. Kim, J. K. Park, E. S. Kang, Y.-T. Kim, C. Heo, O.-S. Lee, S.-G. Kim, W. S. Yun, M. Suh and Y. H. Kim, ACS Nano, 2020, 14, 664-675.

149 F. Xiao, Z. Chen, Z. Wei and L. Tian, Adv. Sci., 2020, 7, 2001048.

150 B. Liu, Y. Wang, Y. Miao, X. Zhang, Z. Fan, G. Singh, X. Zhang, K. Xu, B. Li, Z. Hu and M. Xing, Biomaterials, 2018, 171, 83-96.

151 Y. Ye, Y. Zhang, Y. Chen, X. Han and F. Jiang, Adv. Funct. Mater., 2020, 30, 2003430.

152 Q. Rong, W. Lei and M. Liu, Chem.-Eur. J., 2018, 24, 1693016943.

153 Z. Wang, H. Zhou, W. Chen, Q. Li, B. Yan, X. Jin, A. Ma, H. Liu and W. Zhao, ACS Appl. Mater. Interfaces, 2018, 10, 14045-14054.

154 H. Ji, X. Song, H. Cheng, L. Luo, J. Huang, C. He, J. Yin, W. Zhao, L. Qiu and C. Zhao, ACS Appl. Mater. Interfaces, 2020, 12, 31079-31089. 